This is an author-created, un-copyedited version of an article accepted for publication in 2D Materials. The publisher is not responsible for any errors or omissions in this version of the manuscript or any version derived from it. The Version of Record is available online at https://doi.org/10.1088/2053-1583/4/1/015043.

Work function of graphene multilayers on $\mathrm{SiC}(0001)$

Samir Mammadov, Jürgen Ristein, Julia Krone, Christian Raidel, Martina Wanke, Veit Wiesmann, Florian Speck and Thomas Seyller

2D Materials 4 (2017) 015043 


\title{
Work function of graphene multilayers on $\mathrm{SiC}(0001)$
}

\author{
Samir Mammadov ${ }^{1}$, Jürgen Ristein ${ }^{2}$, Julia Krone ${ }^{1}$, Christian \\ Raidel $^{1}$, Martina Wanke ${ }^{1}$, Veit Wiesmann ${ }^{3}$, Florian Speck ${ }^{1}$ and \\ Thomas Seyller ${ }^{1}$ \\ ${ }^{1}$ Institut für Physik, TU Chemnitz, Reichenhainer Str. 70, 09126 Chemnitz, \\ Germany \\ ${ }^{2}$ Lehrstuhl für Laserphysik, Universität Erlangen-Nürnberg, Staudtstr. 1, 91058 \\ Erlangen, Germany \\ ${ }^{3}$ Fraunhofer Institute for Integrated Circuits IIS, Am Wolfsmantel 33, 91058 \\ Erlangen, Germany \\ E-mail: samir.mammadov@physik.tu-chemnitz.de, \\ thomas.seyller@physik.tu-chemnitz.de
}

\begin{abstract}
The work function and electronic structure of epitaxial graphene as well as of quasi-freestanding graphene multilayer samples were studied by Kelvin probe and angle resolved photoelectron spectroscopy. The work function converges towards the value of graphite as the number of layers is increased. Thereby, n-type doped epitaxial graphene layers have a work function lower than graphite and p-type doped quasi-freestanding graphene layers exhibit a work function higher than graphite. We explain the behaviour by the filling of the $\pi$-bands due to substrate interactions.
\end{abstract}

Keywords: graphene, silicon carbide, work function, doping, ARPES, Kelvin probe

Submitted to: 2D Mater.

\section{Introduction}

Sublimation growth of epitaxial graphene (EG) on silicon carbide ( $\mathrm{SiC}$ ) has evolved to a method of choice for reliable graphene production [1-4]. EG on $\mathrm{SiC}$ has been a subject of intensive research due to various promising applications, such as, e.g., high frequency transistors [5-7], resistance standards [8] and spintronics [9]. A profound knowledge of the basic parameters of the electronic structure and its dependence on substrate and interface modification is a prerequisite for future applications of graphene in carbon-based electronic devices. Yet, such fundamental questions as how the work function of epitaxial graphene varies with the number of layers and how it actually differs from graphite remain scarcely studied systematically [10-15], in the sense that samples prepared under identical and controlled conditions are investigated in a clean environment, i.e. in ultra high vacuum (UHV) using the same analytical tools. 
In this study we experimentally address this issue by making precise Kelvin probe measurements of the work function of EG and quasi-freestanding graphene (QFG), ranging from the pure buffer layer to up to three monolayers for EG and from one to three monolayers for QFG. The band structure of graphene multilayers was measured with angle-resolved photoelectron spectroscopy (ARPES) in order to correlate the work function measurements with the doping level of the samples. The local structure of QFG samples was studied by scanning tunneling microscopy (STM) in order to highlight the differences in substrate coupling of QFG in comparison to previous studies on EG multilayers.

\section{Experimental details}

EG samples used in this study were formed by sublimation growth in argon atmosphere [3] using a custom built reactor [16] on the Si-face of n-type $6 \mathrm{H}-\mathrm{SiC}(0001)$ substrates (bulk doping concentration of $n \approx 5 \times 10^{17} \mathrm{~cm}^{-3}$ ), purchased from SiCrystal AG. By adjusting the temperature and the Ar pressure, the thickness of EG was controlled from the pure buffer layer to up to three monolayers on the buffer layer. The different EG samples are denoted as MLG, BLG, and TLG for one, two, or three layers of graphene on top of the buffer layer, respectively. The buffer layer, denoted $6 \sqrt{3}$ for short, is also known as carbon-rich $(6 \sqrt{3} \times 6 \sqrt{3}) \mathrm{R} 30^{\circ}$ reconstruction. As was discussed previously [17], it is a covalently bound carbon layer which is structurally equivalent to a graphene layer but without the characteristic $\pi$-bands due to the interaction with the substrate.

QFG samples were prepared by annealing EG in ultra-pure hydrogen [18-20] using a custom built setup. This process leads to intercalation of hydrogen between graphene and the $\mathrm{SiC}$ substrate, where hydrogen saturates the $\mathrm{SiC}$ surface, thereby releasing the buffer layer and converting a sample that consists only of the buffer layer into quasi-freestanding monolayer graphene (QFMLG). In the same way, MLG and BLG are converted into quasi-freestanding bilayer graphene (QFBLG) and quasi-freestanding trilayer graphene (QFTLG), respectively. In other words, the conversion of the buffer layer adds one graphene layer to the stack. The sample growth parameters and the precise characterisation of the number of graphene layers by XPS are given in ref. [21].

The local structure of the QFG surfaces was studied by STM. Measurements were carried out using an Omicron LT-STM. The tips were fabricated from a Pt-Ir wire by electrochemical etching. STM images in constant current mode were obtained at tunnelling currents of typically $10 \mathrm{pA}$ at a sample bias of $300 \mathrm{mV}$.

ARPES spectra were collected with the help of a SPECS PHOIBOS 100 2D CCD system operated at beam line UE56/2-PGM1 of the synchrotron radiation source BESSY II. All measurements were carried out at a pressure of $1 \times 10^{-10} \mathrm{mbar}$ or lower, with the sample held at a temperature of $T \approx 80 \mathrm{~K}$.

Work function measurements were carried out by the Kelvin probe method using a custom-built set-up. There, a gold net $2 \mathrm{~mm}$ in diameter with calibrated work function, held at a distance of typically $0.1 \mathrm{~mm}$ above the sample and vibrating vertically at 
$128 \mathrm{~Hz}$ is used as a non-contacting probe. The work function of the gold probe was calibrated by measuring the contact potential difference between the gold probe and a gold sample, where the absolute work function of the gold sample itself was measured using photoelectron YIELD spectroscopy and precisely determined by a Fowler fit [22]. Thus, knowing the absolute work function of the calibrated gold probe, one obtains the absolute work function of the graphene sample by measuring the contact potential difference between the probe and the sample. Note, that sublimation growth of graphene on $\mathrm{SiC}$ frequently leads to the nucleation of additional layers near step edges (see e.g. ref. [3]) and buffer layer inclusions. The relative area of these regions typically amounts to $10 \%$ of the total area. Our macroscopic Kelvin probe setup averages over larger areas of the sample. An estimation of the error caused by the inhomogeneous regions based on the work functions presented below yields a value that is below the error of $0.05 \mathrm{eV}$ quoted in the tables and figures in the following sections.

In addition to the graphene samples, the work function of freshly cleaved highly oriented pyrolitic graphite (HOPG) was measured.

Prior to all the above mentioned measurements, the ex-situ prepared samples were carefully annealed at $300^{\circ} \mathrm{C}$ to remove possible contaminations from exposure to air. Note that a comparative study like the present one can only be carried out when identical preparation techniques and analytical tools are used.

\section{Results and discussion}

\subsection{Local structure of $Q F G$}

Figure 1 shows typical STM images for (a) QFMLG and (b) QFBLG. Here we recall that previous STM studies of $\mathrm{EG}$ on $\mathrm{SiC}(0001)$ have shown a height modulation with an apparent $6 \times 6$ periodicity which is caused by the underlying buffer layer [23-27]. Apparently, such height modulation is not present in both, the QFMLG and the QFBLG samples (see figure 1) due to the absence of the buffer layer between the graphene layers and the $\mathrm{SiC}$ substrate, in agreement with previous reports for QFMLG [28, 29]. It is important to note that very few surface defects or inhomogeneities were visible on QFG samples, even when measured on a larger scale than shown in figure 1. This strongly indicates that the $\mathrm{H}$-intercalation process leads to negligible damage of the graphene sheets which is in agreement with Raman spectroscopy and electronic transport measurements $[30,31]$. Average peak to peak corrugation for QFMLG sample was around $25 \mathrm{pm}$ with RMS roughness of $5.0 \mathrm{pm}$. Figures 1 (c) and (d) display atomically resolved images of the lattice for QFMLG and QFBLG, respectively. In the former case, the hexagonal structure is clearly visible, while in the latter only three out of six atoms per hexagonal ring are visible. This is indicative of A-B stacking as reported previously [32]. The average peak to peak corrugation for QFBLG sample was the same as for QFMLG at around $25 \mathrm{pm}$ with RMS roughness of $7.0 \mathrm{pm}$. 


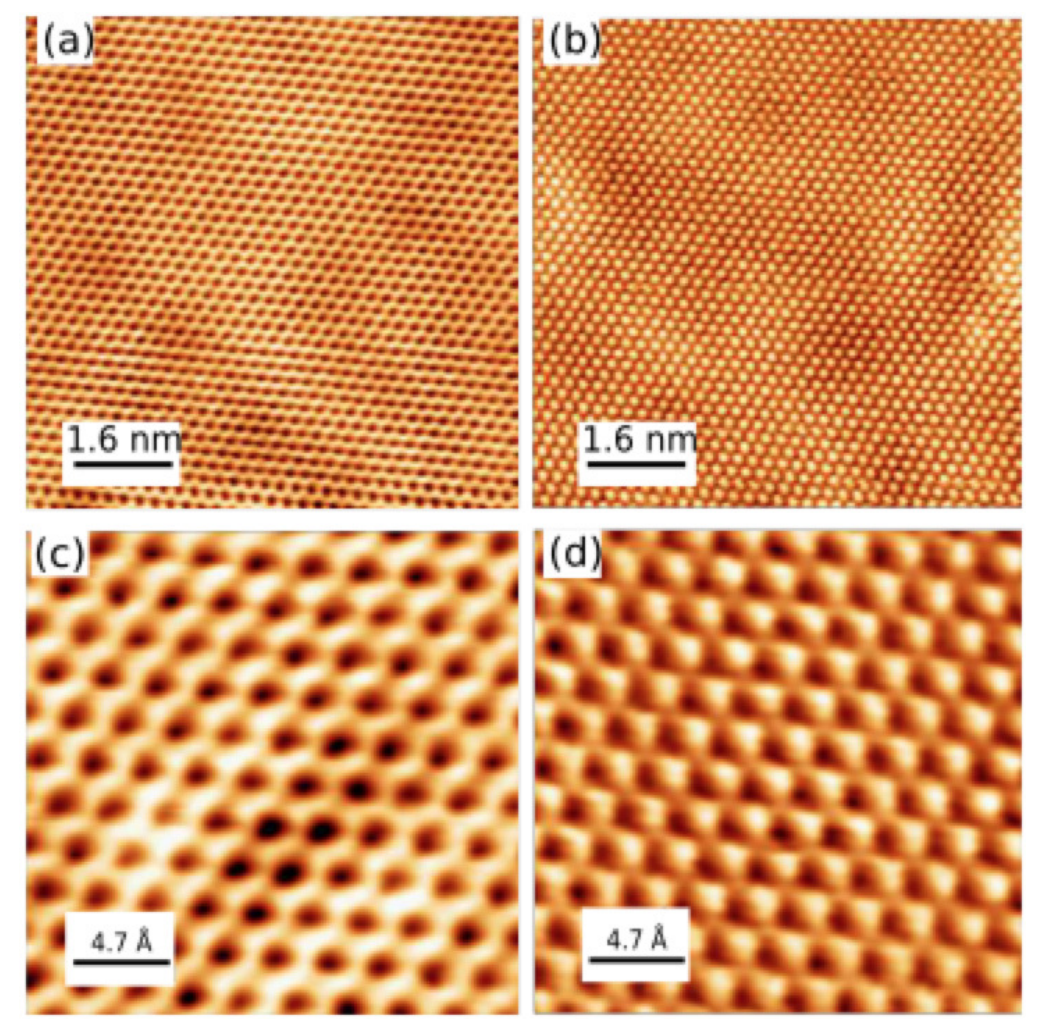

Figure 1. Atomic resolution STM images of (a) QFMLG and (b) QFBLG at $300 \mathrm{mV}$, $10 \mathrm{pA}$. The QFMLG was measured at $T \approx 5 \mathrm{~K}$ and QFBLG at $T \approx 80 \mathrm{~K}$. Enlarged images of QFMLG and QFBLG are shown in (c) and (d), respectively.

\subsection{Work function and band structure}

Figure 2 shows ARPES measurements of QFG and EG samples. As expected, the number of $\pi$-bands increases from one for monolayer to two for bilayer and three for trilayer graphene samples. In order to learn more about the position of the Dirac point $E_{\mathrm{D}}$, the observed bands were fitted with a tight binding model as already used in earlier work $[21,33]$. The Fermi velocity turns out to be $v_{\mathrm{F}}=(0.95 \ldots 1.0) \times 10^{8} \mathrm{~cm} / \mathrm{s}$ and the interlayer hopping matrix element, which is important for bilayers and trilayers, was $\gamma=0.38 \mathrm{eV}$ for both QFBLG and BLG and $\gamma=0.36 \mathrm{eV}$ for QFTLG, in good agreement with previous work $[33,34]$. It has been shown previously that an imbalance in the charge distributed over the two layers of BLG leads to a small bandgap of the order of $0.15 \mathrm{eV}$ [35], in good agreement with $0.12 \mathrm{eV}$ observed here (see figure 2(e)). In the case of QFG, small bandgaps induced by asymmetries in onsite Coulomb potentials are expected but not directly visible in ARPES, which measures only occupied states. However, the presence of a small gap in QFBLG is evident from recent two-photon photoemission study [36]. In the case of QFTLG, we note also the presence of regions of the sample with different stackings as indicated in figure 2 (c). This is not unusual as it was observed previously $[33,34]$. The Dirac point positions obtained from fitting the tight-binding model to the measured $\pi$-bands are compiled in table 1 , together with the 
(a) QFMLG

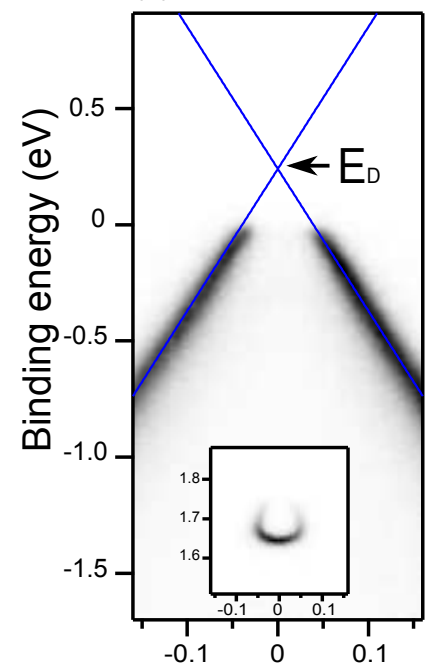

(d) MLG

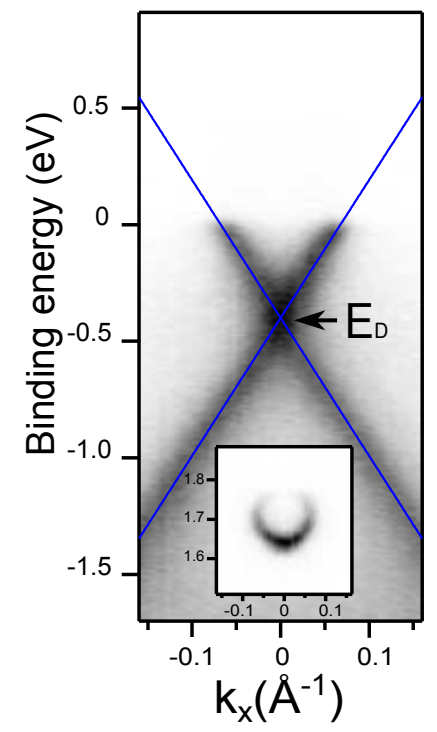

(b) QFBLG

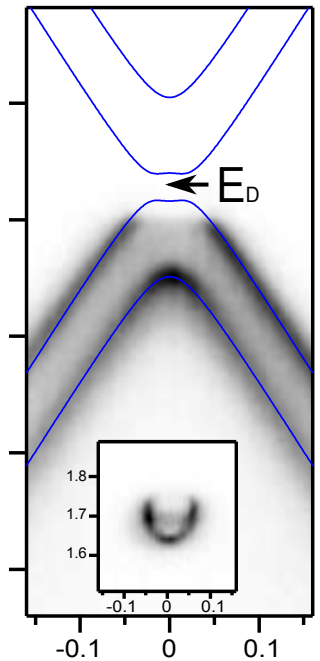

(e) BLG

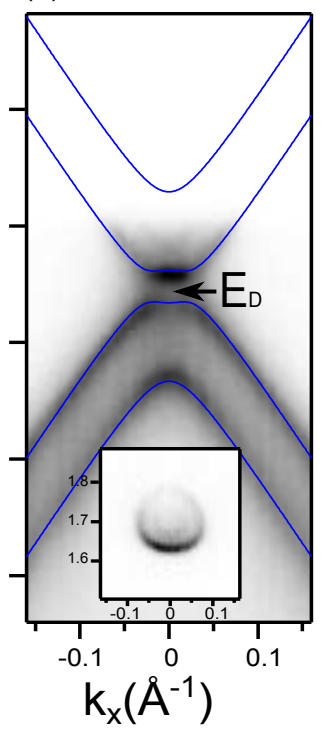

(c) QFTLG
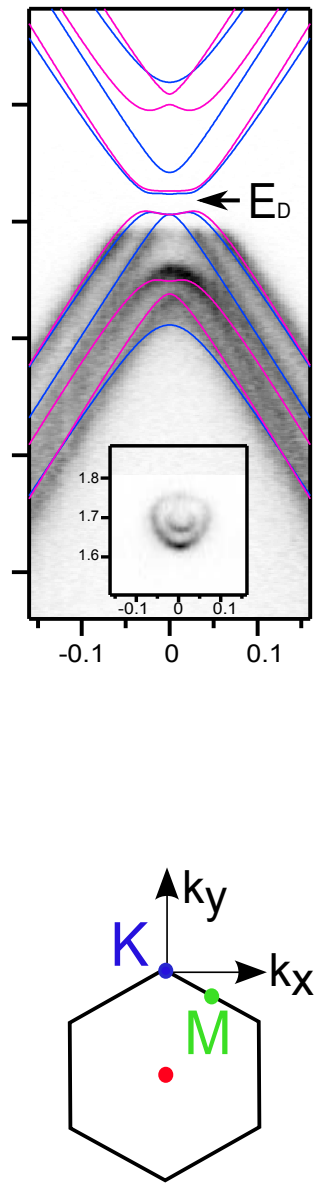

Figure 2. (Color online) $\pi$-bands near $E_{\mathrm{F}}$ for (a) QFMLG, (b) QFBLG, (c) QFTLG, (d) MLG and (e) BLG on n-type $6 \mathrm{H}-\mathrm{SiC}(0001)$. The photon energy was $\hbar \omega=95 \mathrm{eV}$. The blue lines show fitted tight-binding bands. For QFTLG, the red bands correspond to ABC-stacking bands and the blue bands to ABA-stacking. The insets depict the corresponding Fermi surfaces. Schematic representation of graphene in reciprocal space on the right shows directions of $k_{x}$ and $k_{y}$ vectors.

charge carrier concentrations, which were determined from the $\pi$-band Fermi surface areas, which are displayed in the in-sets of figure 2. For comparison available values from the literature are included in that table as well.

As can be seen from table 1, the charge concentration does not change with the number of graphene layers for both, EG and QFG. In order to understand this behaviour, it is important to elaborate on the doping mechanisms in graphene on SiC. As mentioned in previous publications $[21,37,38]$, the doping of graphene on $\mathrm{SiC}$ can 
Table 1. Work functions $\Phi$, Dirac point position $E_{\mathrm{D}}$ with respect to the Fermi level $\left(E_{\mathrm{F}}\right)$, and electron concentration $n$ of $\mathrm{EG}$ and QFG multilayers on n-type $6 \mathrm{H}-\mathrm{SiC}$ substrate.

\begin{tabular}{lccc|ccc}
\hline \multicolumn{3}{c|}{ Experiment } & \multicolumn{3}{c}{ Reference } \\
Samples & $\Phi / \mathrm{eV}$ & $E_{\mathrm{D}} / \mathrm{eV}$ & $n / \mathrm{cm}^{-2}$ & $\Phi / \mathrm{eV}$ & $E_{\mathrm{D}} / \mathrm{eV}$ & $n / \mathrm{cm}^{-2}$ \\
\hline $6 \sqrt{3}$ & $3.89 \pm 0.05$ & & & $3.75[39]$ & & \\
MLG & $4.16 \pm 0.05$ & -0.40 & $1.4 \times 10^{13}$ & $4.05[10]$ & $-0.44[33]$ & $1.1 \times 10^{13}[33]$ \\
BLG & $4.30 \pm 0.05$ & -0.24 & $1.5 \times 10^{13}$ & $4.19[10]$ & $-0.30[33]$ & $1.5 \times 10^{13}[33]$ \\
TLG & $4.43 \pm 0.05$ & & & & $-0.21[33]$ & $1.5 \times 10^{13}[33]$ \\
QFMLG & $4.79 \pm 0.05$ & 0.24 & $-4.2 \times 10^{12}$ & $4.65[10]$ & & \\
QFBLG & $4.71 \pm 0.05$ & 0.14 & $-4.4 \times 10^{12}$ & $4.49[10]$ & & \\
QFTLG & $4.63 \pm 0.05$ & 0.07 & $-4.9 \times 10^{12}$ & & & \\
HOPG & $4.55 \pm 0.05$ & & $4.48[40]$ & & \\
\hline
\end{tabular}

be explained mainly by three different mechanisms. First and relevant only for EG, the dangling bond-like interface states originating from the buffer layer cause an electron transfer to the graphene layers on top. This leads to the high electron concentration of $10^{13} \mathrm{~cm}^{-2}$ in EG samples. Second, the spontaneous polarization of the hexagonal $\mathrm{SiC}$ substrate $[21,37]$ induces hole doping in graphene on $\mathrm{SiC}(0001)$. Once the interface is passivated by hydrogen, this contribution becomes the dominant one, leading to the observed p-type doping of QFG. The third and smallest contribution is caused by the formation of a space charge region in the $\mathrm{SiC}$ substrate in terms of the Schottky model [21]. As all these three mechanisms are caused by the interface and the substrate, which do not change as additional layers build up, there is no reason why the total charge carrier concentration should change for multilayers.

In addition to the Dirac point energy $E_{\mathrm{D}}$ and charge carrier concentration $n$, table 1 also lists the work function of the various samples, which were determined after annealing. The $6 \sqrt{3}$ reconstructed $6 \mathrm{H}-\mathrm{SiC}(0001)$ surfaces showed a work function of $\sim 4.8 \mathrm{eV}$ before annealing, which changed gradually upon annealing, stabilising at $3.89 \mathrm{eV}$. This indicates that adsorbates are likely to adhere to the buffer layer forming a dipole layer and changing the work function. For MLG and BLG samples, a work function of $\sim 4.9 \mathrm{eV}$ was measured before annealing, which suggests that adsorbates are sticking to these surfaces as well. In both cases these adsorbates can be desorbed by annealing at $300^{\circ} \mathrm{C}$, leading to work functions listed in the table. The absolute values of the work function for EG samples are in good agreement with Gugel et al. [10] and the difference in work function between MLG and BLG of $0.14 \mathrm{eV}$ matches the measurements of Filleter et al. [11], although the difference between the buffer layer and MLG is slightly smaller in their work, which can be due to the different annealing conditions. Furhermore, Hibino et al. [14] determined the work function of EG by analysing the secondary electron cutoff in photoelectron emission microscopy experiments. Their results are - with the exception of an offset of around $0.1 \mathrm{eV}$ which might be due to 
different calibration - in good agreement with our values.

Almost no difference before and after annealing was observed for QFG samples. This may indicate an inertness of quasi-freestanding graphene to adsorbates from the air due to its reduced roughness that was witnessed by the STM measurements discussed above. On the other hand, it would also be possible that adsorbates are present but do not lead to the formation of a dipole layer due to different alignments of electronic levels. This question needs to be addressed in future studies. The absolute work functions for QFG samples differ somewhat from those obtained by Gugel et al. [10]. Nevertheless, they observed the same trend. The systematic deviation for both QFG and EG by approximately $0.1 \mathrm{eV}$ points towards differences in the calibration of the experiments.

Melios et al. $[12,13]$ employed Kelvin probe force microscopy to study the work function of EG and QFG. For EG, the convergence of the work function towards that of graphite as the number of layers increases (see below), reported also by Gugel et al. and Hibino et al., is observed in one work of Melios et al. [13], but not in the other [12]. For QFG, convergence to the value of HOPG is not reported by Melios et al. $[12,13]$. This discrepancy could be due to different sample preparation conditions or a contamination of the surfaces because they did not measure in UHV.

Finally, the dependence of the work function on the number of layers is discussed. To that end, the work functions from table 1 are plotted in figure 3 as a function of the number of layers. In addition, $E_{\mathrm{D}}-E_{\mathrm{F}}(\mathrm{EG})$ for epitaxial graphene and $E_{\mathrm{D}}-E_{\mathrm{F}}(\mathrm{QFG})$ for quasi-freestanding graphene samples, taken from the ARPES measurements, are plotted in figure 3. While the work function of EG increases with the number of layers, the work function of QFG decreases and both converge to the value of HOPG. This can be explained as follows. The total areal charge density provided by the three different doping mechanisms, which have been alluded to above, has to be stored in the stack of graphene layers and distributed amongst the layers. Thereby two boundary conditions must be met: (i) a reduced charge density towards the outer layers following Poisson's equation and (ii) the requirement of a constant Fermi level. As a consequence, a band bending potential across the stack of graphene layers evolves that brings the surface Fermi level closer and closer towards the intrinsic Fermi level when the number of graphene layers increases. This effect is well represented in figure 3, where the absolute work function values of graphene multilayers follow the energy difference between the Fermi level $E_{\mathrm{F}}$ and the Dirac point $E_{\mathrm{D}}$ and thus reflect the aforementioned changes in the band profiles.

\section{Conclusion}

In summary, we have presented a systematic study of the work function of EG and QFG on $\mathrm{SiC}(0001)$. We observed that the work function converges to that of graphite as the number of layers increased. Additional measurements of the Dirac point energy by ARPES showed that the difference of the work function between EG/QFG and the work function of graphite can be explained by the filling of the $\pi$-bands 


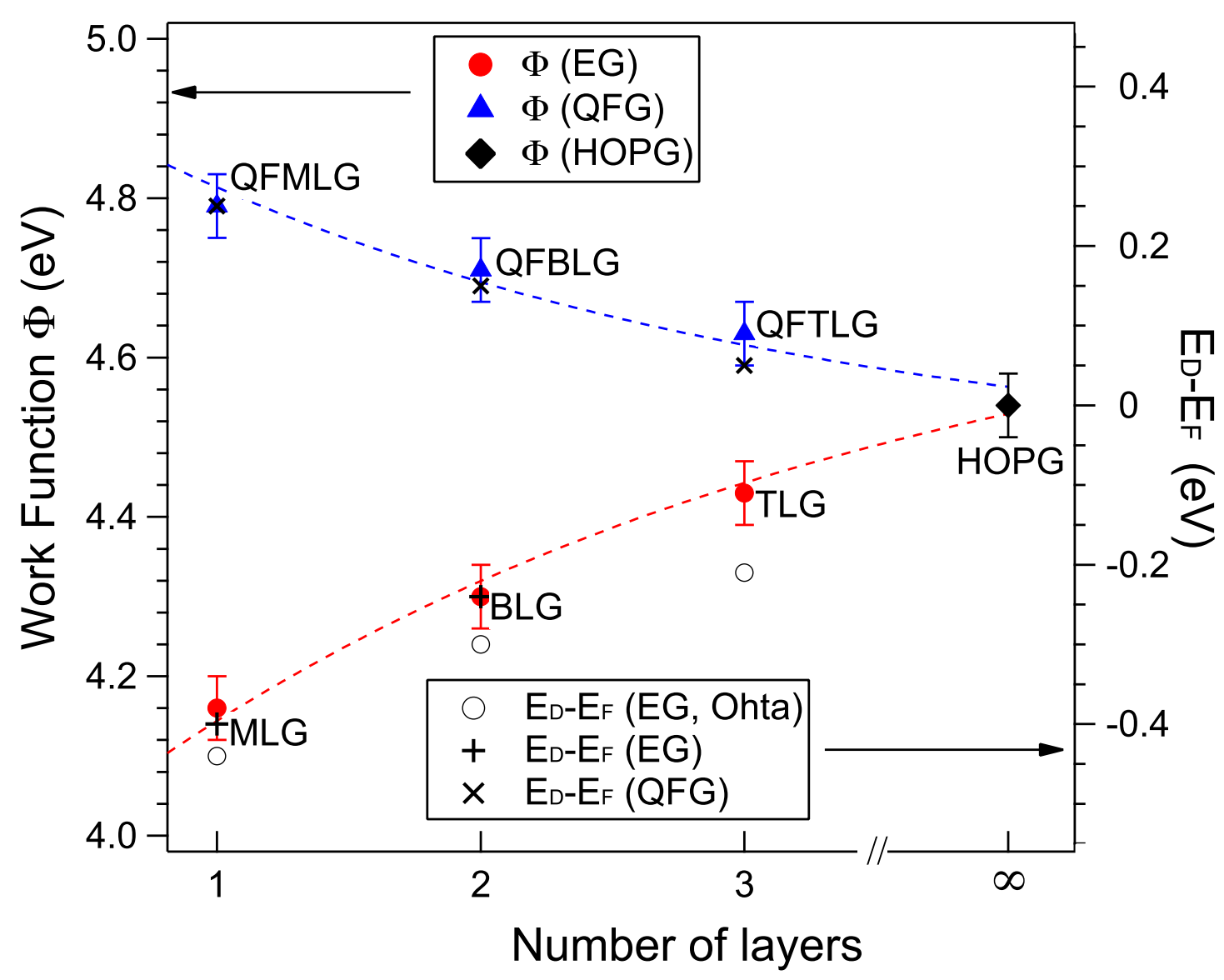

with the electrons/holes by the mechanisms described above, following the relation $\Phi-\Phi_{\mathrm{HOPG}}=E_{\mathrm{D}}-E_{\mathrm{F}}$. This relation also indicates that the work funciton of neutral graphene is the same as that of graphite.

\section{Acknowledgement}

The authors are grateful to Karsten Horn, Hendrik Vita and Stefan Böttcher from the Fritz-Haber-Institute for their ongoing support during beam times at BESSY II as well as for fruitful discussions. The research leading to these results has received partial funding from the European Union Seventh Framework Programme under grant agreement n604391 Graphene Flagship and from the DFG within the Collaborative Research Centre SFB 953 Synthetic Carbon Allotropes and the Priority Programme SPP 1459 Graphene. 
Work function of graphene multilayers on SiC(0001)

\section{References}

[1] Berger C et al 2006 Science 312 1191-6

[2] Virojanadara C, Syväjarvi M, Yakimova R, Johansson L I, Zakharov A A and Balasubramanian T 2008 Phys. Rev. B 78245403

[3] Emtsev K V et al 2009 Nature Mater. 8 203-7

[4] First P N, de Heer W A, Seyller T, Berger C, Stroscio J A and Moon J-S 2010 MRS Bull. 35 296-305

[5] Moon J S et al 2009 IEEE Electron Device Lett. 30 650-2

[6] Lin Y-M, Dimitrakopoulos C, Jenkins K A, Farmer D B, Chiu H-Y, Grill A and Avouris P 2010 Science $\mathbf{3 2 7} 662$

[7] Wu Y et al 2012 Nano Lett. 12 3062-7

[8] Tzalenchuk A, Lara-Avila S, Kalaboukhov A, Paolillo S, Syväjärvi M, Yakimova R, Kazakova O, Janssen T J B M, Fal'ko V and Kubatkin S 2010 Nature Nanotech. 5 186-9

[9] Maassen T, van den Berg J J, IJbema N, Fromm F, Seyller T, Yakimova R and van Wees B J 2012 Nano Lett. 12 1498-502

[10] Gugel D, Niesner D, Eickhoff C, Wagner S, Weinelt M and Fauster T 2015 2D Mater. 2045001

[11] Filleter T, Emtsev K V, Seyller T and Bennewitz R 2008 Appl. Phys. Lett. 93133117

[12] Melios C, Panchal V, Giusca C E, Strupiński W, Silva S R P and Kazakova O 2015 Sci. Rep. 5 10505

[13] Melios C, Spencer S, Shard A, Strupiński W, Silva S R P and Kazakova O 2016 2D Mater. 3 025023

[14] Hibino H, Kageshima H, Kotsugi M, Maeda F, Guo F-Z and Watanabe Y 2009 Phys. Rev. B 79 125437

[15] Panchal V, Pearce R, Yakimova R, Tzanenchuk A, Kazakova O 2013 Sci. Rep. 32597

[16] Ostler M, Speck F, Gick M and Seyller T 2010 Phys. Status Solidi B 247 2924-6

[17] Emtsev K V, Speck F, Seyller T, Ley L and Riley J D 2008 Phys. Rev. B 77155303

[18] Riedl C, Coletti C, Iwasaki T, Zakharov A A and Starke U 2009 Phys. Rev. Lett. 103246804

[19] Riedl C, Coletti C, Iwasaki T and Starke U 2010 Mater. Sci. Forum 645-648 623-8

[20] Speck F, Ostler M, Röhrl J, Jobst J, Waldmann D, Hundhausen M, Ley L, Weber H B and Seyller T 2010 Mater. Sci. Forum 645-648 629-32

[21] Mammadov S, Ristein J, Koch R J, Ostler M, Raidel C, Wanke M, Vasiliauskas R, Yakimova R and Seyller T 2014 2D Mater. 1035003

[22] Fowler R H 1931 Phys. Rev. 38 45-56

[23] Mallet P, Varchon F, Naud C, Magaud L, Berger C and Veuillen J-Y 2007 Phys. Rev. B 76 041403(R)

[24] Rutter G M, Crain J N, Guisinger N P, Li T, First P N and Stroscio J A 2007 Science 317 219-22

[25] Brar V W, Zhang Y, Yayon Y, Ohta T, McChesney J L, Bostwick A, Rotenberg E, Horn K and Crommie M F 2007 Appl. Phys. Lett. 91122102

[26] Lauffer P, Emtsev K V, Graupner R, Seyller T, Ley L, Reshanov S A and Weber H B 2008 Phys. Rev. B 77155426

[27] Riedl C, Starke U, Bernhardt J, Franke M and Heinz K 2007 Phys. Rev. B 76245406

[28] Goler S et al 2013 Carbon 51 249-54

[29] Murata Y, Mashoff T, Takamura M, Tanabe S, Hibino H, Beltram F and Heun S 2014 Appl. Phys. Lett. 105221604

[30] Speck F, Jobst J, Fromm F, Ostler M, Waldmann D, Hundhausen M, Weber H B and Seyller T 2011 Appl. Phys. Lett. 99122106

[31] Fromm F, Oliveira Jr M H, Molina-Sánchez A, Hundhausen M, Lopes J M J, Riechert H, Wirtz L and Seyller T 2013 New J. Phys. 15043031

[32] Stolyarova E, Rim K T, Ryu S, Maultzsch J, Kim P, Brus L E, Heinz T F, Hybertsen M S and Flynn G W 2007 Proc. Natl. Acad. Sci. U.S.A. 104 9209-12 
[33] Ohta T, Bostwick A, McChesney J L, Seyller T, Horn K and Rotenberg E 2007 Phys. Rev. Lett. 98206802

[34] Coletti C et al 2013 Phys. Rev. B 88155439

[35] Ohta T, Bostwick A, Seyller T, Horn K and Rotenberg E 2006 Science 313 951-4

[36] Ulstrup S et al 2014 Phys. Rev. Lett. 112257401

[37] Ristein J, Mammadov S and Seyller T 2012 Phys. Rev. Lett. 108246104

[38] Kopylov S, Tzalenchuk A, Kubatkin S and Fal'ko V I 2010 Appl. Phys. Lett. 97112109

[39] Mattausch A and Pankratov O 2007 Phys. Rev. Lett. 99076802

[40] Hansen W N and Hansen G J 2001 Surf. Sci. 481 172-84 